\title{
LCoS-SLM technology based on Digital Electro-optics Platform and using in dynamic optics for application development
}

Chun-Wei Tsai, Chen Wang, Bo-Han Lyu, Chen-Hsien Chu

Chun-Wei Tsai, Chen Wang, Bo-Han Lyu, Chen-Hsien Chu, "LCoS-SLM technology based on Digital Electro-optics Platform and using in dynamic optics for application development," Proc. SPIE 10452, 14th Conference on Education and Training in Optics and Photonics: ETOP 2017, 104523Q (16 August 2017); doi: 10.1117/12.2257048

SPIE Event: 14th Conference on Education and Training in Optics and Photonics, ETOP 2017, 2017, Hangzhou, China 


\title{
LCoS-SLM Technology Based on Digital Electro-optics Platform and Using in Dynamic Optics for Application Development
}

\author{
Chun-Wei Tsai*, Chen Wang, Bo-Han Lyu, and Chen-Hsien Chu \\ Jasper Display Corp. (JDC), 7F-16, No. 81, Shui-Li Rd., Hsinchu 30059, Taiwan
}

\begin{abstract}
Digital Electro-optics Platform is the main concept of Jasper Display Corp. (JDC) to develop various applications. These applications are based on our X-on-Silicon technologies, for example, X-on-Silicon technologies could be used on Liquid Crystal on Silicon (LCoS), Micro Light-Emitting Diode on Silicon ( $\mu$ LEDoS), Organic Light-Emitting Diode on Silicon (OLEDoS), and Cell on Silicon (CELLoS), etc. LCoS technology is applied to Spatial Light Modulator (SLM), Dynamic Optics, Wavelength Selective Switch (WSS), Holographic Display, Microscopy, Bio-tech, 3D Printing and Adaptive Optics, etc. In addition, $\mu$ LEDoS technology is applied to Augmented Reality (AR), Head Up Display (HUD), Head-mounted Display (HMD), and Wearable Devices. Liquid Crystal on Silicon - Spatial Light Modulator (LCoSSLM) based on JDC's On-Silicon technology for both amplitude and phase modulation, have an expanding role in several optical areas where light control on a pixel-by-pixel basis is critical for optimum system performance. Combination of the advantage of hardware and software, we can establish a "dynamic optics" for the above applications or more. Moreover, through the software operation, we can control the light more flexible and easily as programmable light processor.
\end{abstract}

Keywords: Digital Electro-optics Platform, On-Silicon, Liquid Crystal on Silicon (LCoS), Spatial Light Modulator (SLM), Micro-LED on Silicon ( $\mu$ LEDoS), Dynamic Optics, Education Kit (EDK), Optics Education

\section{INTRODUCTION}

Taiwan has strong supply chain for silicon industry. Based on the accumulation of strength on both electronics and optics in Taiwan, the Digital Electro-optics Platform we proposed could derive many application for many fields, such as wearable, home, automobile, entertainment, medical treatment, and food. In semiconductor, there is a famous law to describe the progress for semiconductor process. It is Moore's law. Right now the barrier for mass production is $10 \mathrm{~nm}$. We could continue walking on technical path to overcome $10 \mathrm{~nm}, 7 \mathrm{~nm}$, even $5 \mathrm{~nm}$. But there is a different path, application path. On this path, we could choose the branch for electronics, which continues to grow, or we could choose the branch for electro-optics. I also call it as "X-on silicon". On this path, new elements, e.g. optics and material, are added into silicon industry. I think this could maximize the opportunities. Light technology would be the key to grasp the opportunity. Just as the proclamation of the UN, "Light-based technology is a major economic driver with potential to revolutionize the $21^{\text {st }}$ century [1]". At this moment, it is necessary to find out the self-position and confirm the development direction quickly. With multi-party cooperation, the cornerstone of the industry could be raised and the time length of technology development could be folded up. Therefore, it would have a chance to grasp the world level market. Besides, education and training must also act simultaneously. This can provide the industry adequate engineering and R\&D people. SLM is a useful and powerful optical instrument to modulate the optical wavefront and has been widely used on various applications. LCoS-SLM is capable of accepting a video signal and converting it into a holographic image. The video signal, also called computer generated holograms (CGH), is one method to generate holographic interference patterns digitally. Moreover, there are three steps to generate CGH, such as computing the virtual scattered wavefront, encoding the interference pattern, and reconstructing the holographic image. Due to the precision wavefront control, LCoS-SLM could be used on dynamic optics technology [2]. The applications of dynamic optics include beam shaping [3], beam steering [4], diffractive optical element (DOE) [5], optical manipulation [6], orbital angular momentum (OAM) [7], etc.

*chunwei.tsai@jasperdisplay.com; phone 886-3-575-2768; fax 886-3-575-2628; www.jasperdisplay.com

14th Conference on Education and Training in Optics and Photonics: ETOP 2017, edited by Xu Liu,

Xi-Cheng Zhang, Proc. of SPIE Vol. 10452, 104523Q - @ 2017 ICO, IEEE, OSA, SPIE

CCC code: $0277-786 X / 17 / \$ 18 \cdot$ doi: $10.1117 / 12.2257048$

Proc. of SPIE Vol. 10452 104523Q-1 


\subsection{Digital Electronic-optics Platform (X-on Silicon)}

The core technology of Digital Electro-optics Platform (X-on Silicon) includes the highest pixel density approaching, the smallest pixel pitch, and the digital driving technology. Our mission is to lead an Ecosystem for various applications based on our X-on Silicon technologies, for example, X-on-Silicon technologies could be used on LCoS, $\mu$ LEDoS, OLEDoS, and CELLoS, etc. There are three principal elements in our provided winning strategy, such as innovation domain know-how, and Digital Electro-optics Platform. Innovation is the key to competitive advantage. Domain knowhow is the core to go deep and to differentiate for enterprises. Regarding digital Electro-optics Platform, it would be a powerful tool to develop technology and product for startups and enterprises. Figure 1 (a) show the Ecosystem based on digital-optics platform (X-on Silicon). Regarding the support of technologies and services from JDC, we can support the hardware and software of backplane and LCoS-SLM. Even this kind of support is included custom service and MiniFAB. The objective of us is to help our customers and our partners to develop their fantastic applications as soon as possible. The photo of digital Electro-optics Platform (X-on Silicon) is shown as Figure 1 (b). Three-dimension holographic display based on LCoS-SLM technology and three color microdisplay based on $\mu$ LEDoS is demonstrated as Figure 1 (c) and Figure 1 (d), respectively.

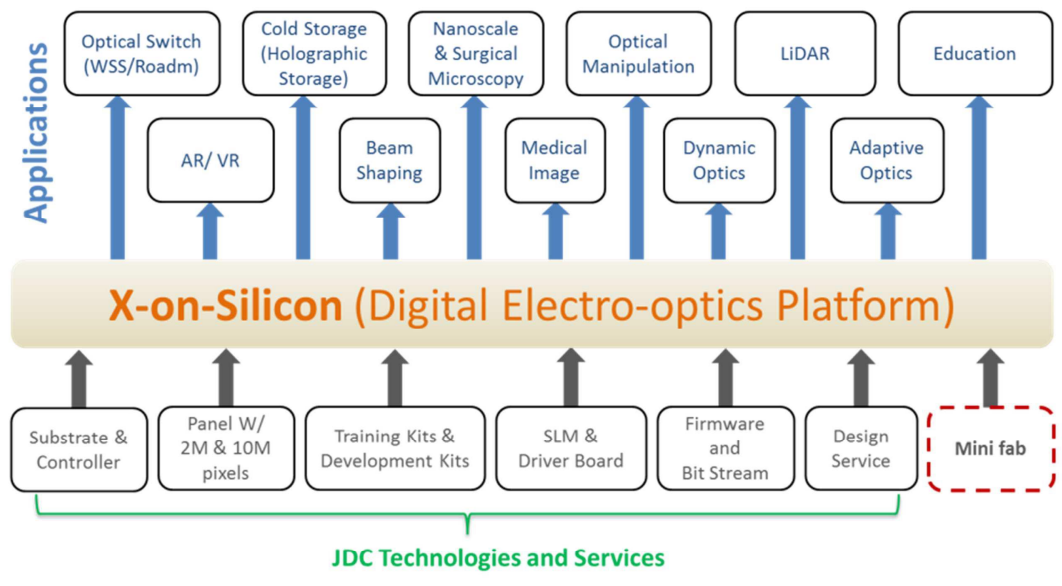

(a)

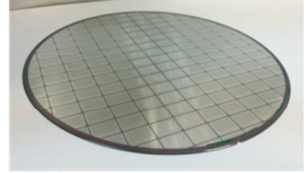

(b)

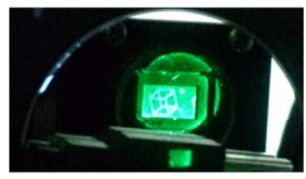

(c)

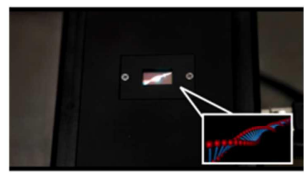

(d)

Figure 1. (a) Various application based on Digital Electro-optics Platform (X-on Silicon), (b) Digital Electro-optics Platform, (c) 3D holographic display based on LCoS-SLM, and (d) 3 color microdisplay based on $\mu$ LEDoS.

\subsection{Liquid Crystal on Silicon (LCoS)}

LCoS technology is one example by using the Digital Electro-optics Platform. Regarding the LCoS technology, the liquid crystal molecules would be twisted by adding different voltage. Through the characteristic of liquid crystal, the incident coherent light source has variation of phase retardation when the coherent light source passes through the liquid crystal layer. LCoS could be regarded as spatial light modulator (SLM) to implement the amplitude modulation and the phase modulation. Figure 2 (a) shows the architecture and photo of reflective type LCoS-SLM. Moreover, LCoS-SLM has the properties of smaller pixels, higher aperture ratio, and thinner cell gap. Based on these properties, LCoS-SLM also has the advantages of higher diffraction angle, higher efficiency, ad faster response time. Recently, LCoS-SLM has been widely used on various applications, such as dynamic optics, adaptive optics [8-9], holographic display [10], microscopy [11], Bio-tech, beam shaping, beam steering, wavelength selective switch (WSS) [12], diffraction optical element (DOE), semiconductor equipment [13], and 3D metrology [14]. LCoS-SLMs, based on JDC's On-Silicon technology for both amplitude and phase modulation, have an expanding role in several optical areas where light control on a pixel-by-pixel basis is critical for optimum system performance. We combine the advantage of hardware and software, and we can establish a "dynamic optics" for the above applications or more. Moreover, through the software operation, we can control the light more flexible and easily as programmable light processor. There are two diffraction images at different focal plane as demonstrated as Figure 2 (b). 


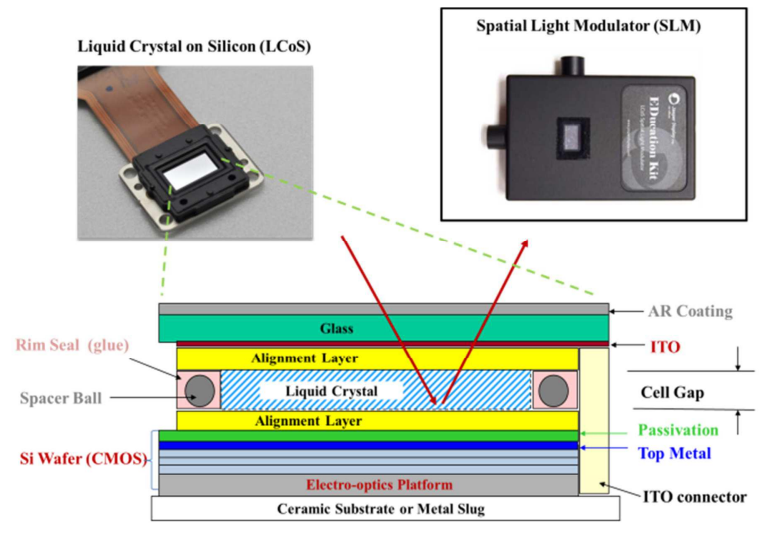

(a)
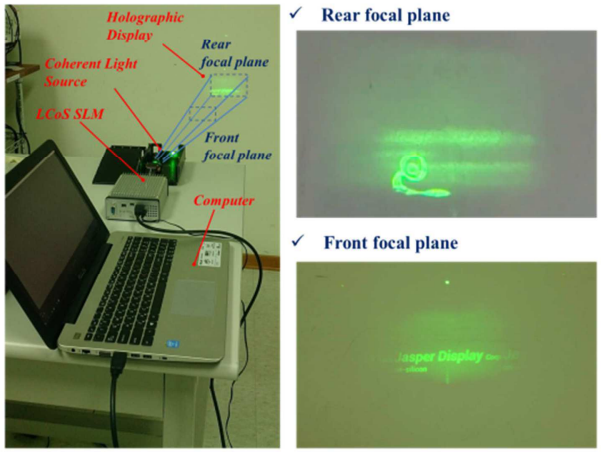

(b)

Figure 2. (a) The architecture and product photo of reflective type LCoS-SLM, and (b) Two diffraction images are demonstrated at different focal plane.

\subsection{Micro LED on Silicon $(\mu \mathrm{LEDoS})$}

$\mu \mathrm{LEDoS}$ technology is another example by using the Digital Electro-optics Platform (X-on-Silicon). Regarding the $\mu \mathrm{LEDoS}$, our megapixel and digital electro-optics platform could provide the active matrix driving and digital modulation on each Micro-LED. Based on these advantages, $\mu$ LEDoS technology [15-16] is applied to Augmented Reality (AR), Virtual Reality (VR), Head Up Display (HUD), Head-mounted Display (HMD), and Wearable Devices. One of our partner, glō, has been successfully demonstrated that three color micro-LEDs could be driven and be enhanced the performance by using JDC's digital electro-optics platform [17]. glō's nanowire LEDs enabling RGB monolithic chip integration [18]. The actual nanowire LEDs include blue, green, and red as shown as Figure 3 (a). The arrangement and switch method of nanowire LEDs is shown as Figure 3 (b). SEM of actual nanowire chip and the efficiency vs. chip size are shown as Figure 3 (c) and Figure 3 (d), respectively.

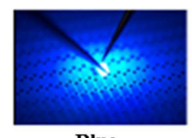

Blue

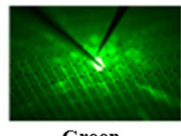

(a)
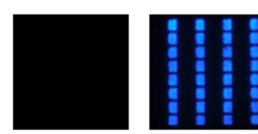

off

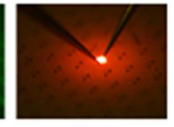

Red

(a)

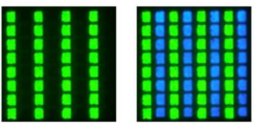

Green $\rightarrow$ Blue+Green

(b)

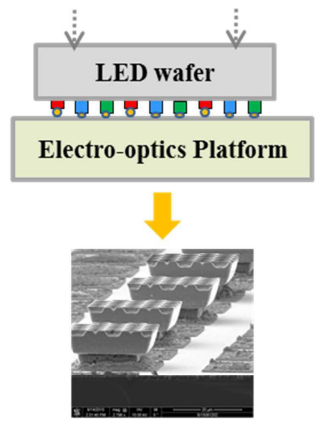

(c)

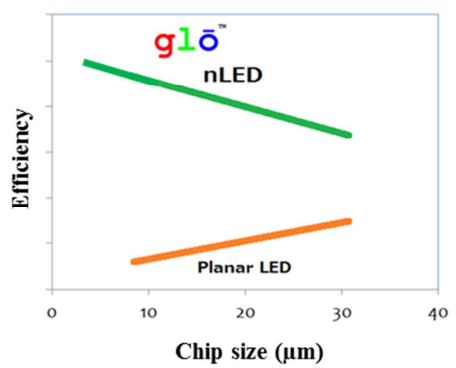

(d)

Figure 3. (a) Photo of actual nanowire LEDs, (b) The arrangement and switch method of nanowire LEDs, (c) SEM of actual nanowire chip, and (d) The efficiency vs. chip size based on glō's nanowire LEDs.

\subsection{MiniFAB Platform}

Richly cultivating education, JDC also do our maximum effort to build a MiniFAB Platform as a bridge to cooperate with academia, research institutions, and industries. Figure 4 (a) shows a one example of liquid crystal industry chain that include MiniFAB concept. Regarding MiniFAB, it is a way to satisfy a special market for small volumes and for very special requirement. We look for the possible partners who have expertise to develop the corresponding recipes based on our Digital Electro-optics Platform (X-on Silicon). The recipes could be any possible material, such as liquid crystal, micro light-emitting diode, or organic light-emitting diode, cells, microfluidics, or other material. Hence, we 
welcome all researcher and expert to join this kind of cooperation to turn the research and development into seeds of innovation and startup. By the complementary, a complete LCoS industry chain will be build up. We consider MiniFAB as the bridge which connects the supply side and the demand side. Not only to deepen the technologies, but also to expand the industry of light. In fact, there are many companies integrated LCoS-SLM or Digital Electro-optics Platform (X-on Silicon) technology into their products already, especially bio-medical field. Recently, the topic is active to integrate the light technology into more and more technologies. Indeed, many new products and technologies emerge by this kind of combination, so we are confident that our LCoS-SLM or Digital Electro-optics Platform would be able to bring more opportunities for light industry.

In Taiwan, one alliance named "Things On Silicon Alliance (TOSA)" was established to support X-on silicon ECOsystem. Its main functions include education, market development, infrastructure (MiniFAB), and promotion for vertical application. Figure 4 (b) shows a novel cooperation model between TOSA, JDC, academia, research institutions, and industries. JDC provide the Digital Electro-optics Platform (X-on Silicon) and digital driving method. Then, we encourage the academia, research institution, and industries to setup a MiniFAB and to cooperate with us. Based on the innovation and domain know-how from the possible experts, we can enable the new platform to support the industrial in the near future. However, through this kind cooperation model, we look forward to create new concepts, new approaches, new technologies, new materials, and new application with our partners.

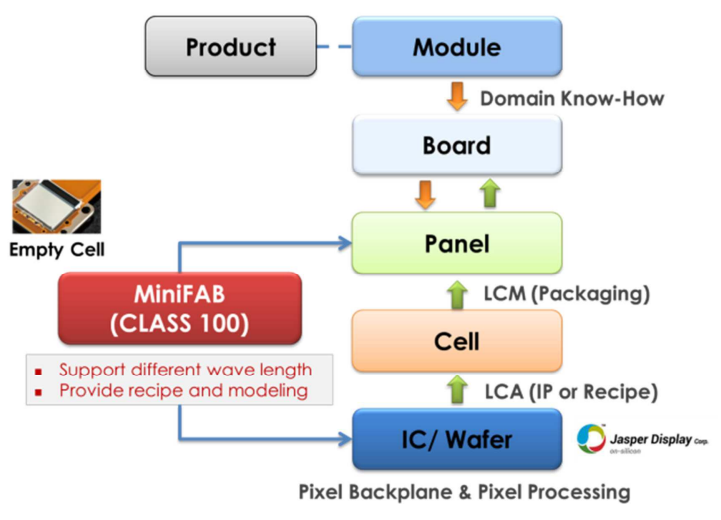

(a)

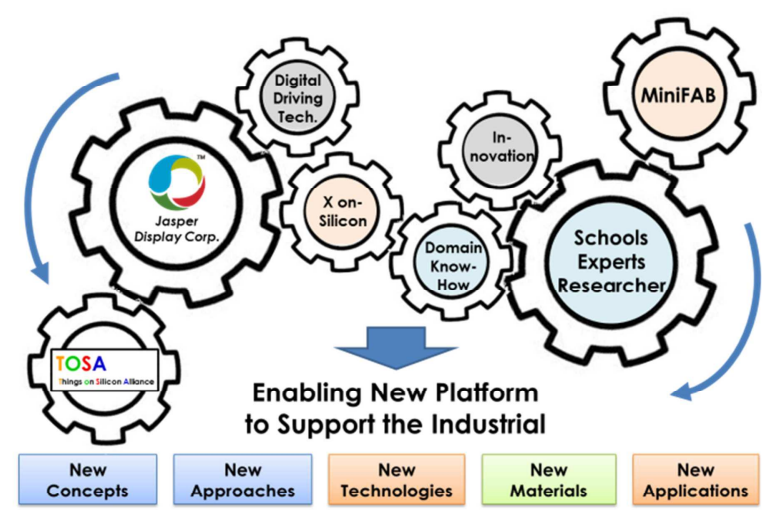

(b)

Figure 4. (a) Liquid crystal industry chain that include MiniFAB concept, and (b) Novel cooperation model between TOSA, JDC, academia, research institutions, and industries.

\section{SLM DEVELOPMENT KIT (SDK) AND EDUCATION KIT (EDK)}

JDC's SLM Development Kits (SDK) targets diverse applications, ranging from consumer and mobile projectors, professional projectors, fringe projectors for 3D digitizers, diffractive optical elements for "structured light" applications, etc. Our LCoS-SLM devices could be used with an associated controller ASIC and evaluation modulation software. Regarding the Education Kits (EDK), it provides a training platform for university students to develop skills and spur innovations for future optoelectronic applications. EDK can be configured to do different electro-optical and photonic experiments, such as the pixelated structure, amplitude modulation, phase modulation, wavefront modulation, diffraction and interference, the Michelson interferometer, dispersion, signal processing, Talbot image, and phase-shifting digital holography. We realized one detail that optical technology must be rooted in education to establish a solid foundation. How to find a balance between theory and practice in optics education is our mission and target. Through the experiments, students become more familiar with the principles of optics, and they may find more details during experiments. This would be a great help to technology and product development when they enter industries in the future. We think that the traditional optical experiment equipment is not enough currently. In the generation of hardwaresoftware combination, an important concept about "dynamic optics" should be established. It means we could use software to control the light. That is why we must develop "Education Kit (EDK)" for education field. We believe that a solid optical education and training system can provide the industry adequate engineering and R \& D people. This is very 
important to the whole eco-system of light industry. Figure 5 (a) shows the comparison between the traditional optics based on lenses and dynamic optics based on LCoS-SLM. LCoS-SLM could provide a program-controllable liquid crystal array for dynamic optics. It dynamically changes the amplitude or phase of light and gives the obvious advantage, "Flexibility," to the system. In addition, LCoS-SLM also have several advantages such as simple operation, time saving, space saving, cost saving, and more function. The Graphic User Interface (GUI) of LCoS-SLM controller and microdisplay are shown in Figure 5 (b). The dynamic CGH phase pattern and the dynamic diffraction images of grating phase, vortex phase, and axicon phase are shown in Figure 5 (c). These dynamic diffraction images are generated by different CGH phase pattern. The function of these dynamic phase patterns are similar as the diffractive optical element (DOE).

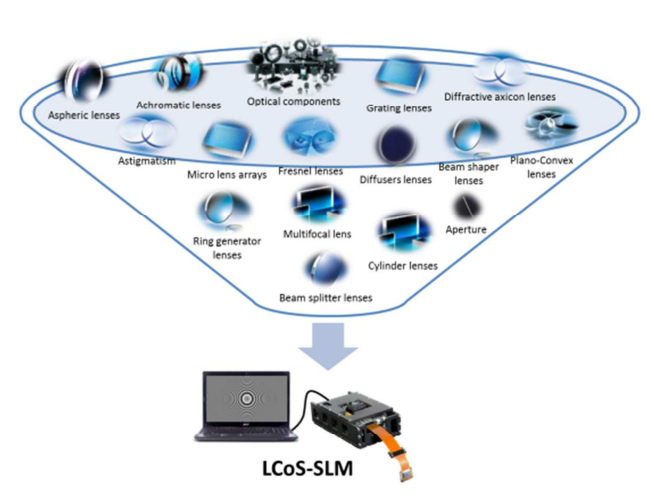

(a)

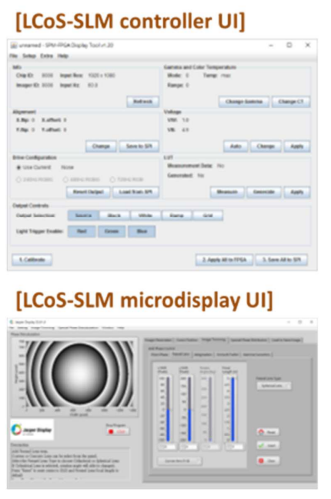

(b)

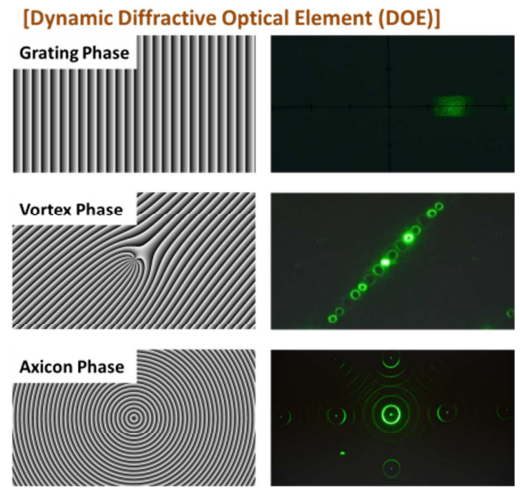

(c)

Figure 5. (a) Comparison between traditional optics based on lenses and dynamic optics based on LCoS-SLM, (b) GUI of LCoS-SLM controller and microdisplay, and (c) Dynamic CGH phase pattern and dynamic diffraction images.

\section{EXPERIMENT SETUP AND MEASUREMENT RESULTS}

Multi-function and easy-to-use modulation software that we have already developed was based on LabVIEW system, such as CGH modulation software [19] and EDK experiment software [20-21]. There are mainly four functions in CGH modulation software, such as CGH generation, CGH reconstruction, image trimming, and special phase distribution. Regarding the EDK experiment software, there are mainly three topics in our EDK training program and experiment course. These basic experiments include the pixelated structure of LCOS-SLM, amplitude modulation, phase modulation, wavefront modulation, diffraction and interference, Michelson interferometer, signal processing, Talbot image, and phase-shift digital holography. However, we believe that a robust LCoS-SLM, operation software, simulation software, training system, and training course can help students to study the fundamental optics, wave optics, and Fourier optics more easily. Based on the above development of CGH modulation software and EDK experiment software, we could enhance the performance of LCoS-SLM as similar as the DOE and use it on various dynamic optics applications. The GUI of LabVIEW based CGH modulation software and the EDK experiment software are shown in Figure 6 (a) and Figure $6(\mathrm{~b})$, respectively.

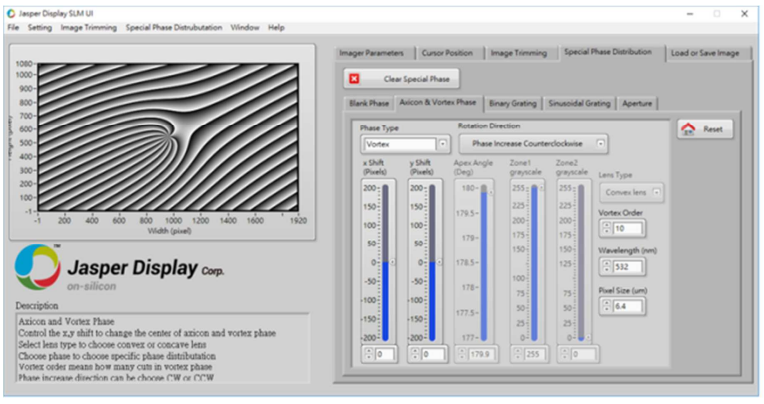

(a)

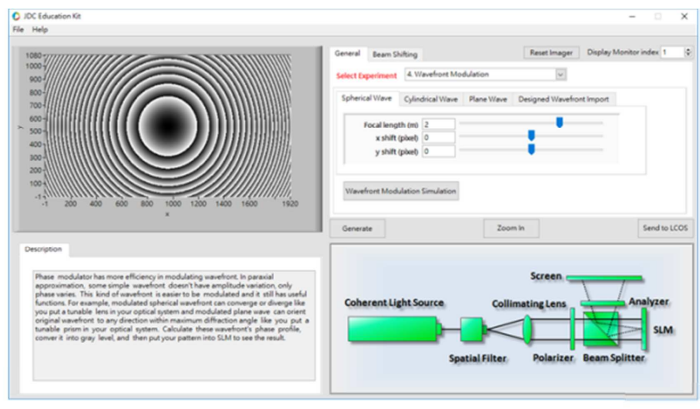

(b)

Figure 6. (a) GUI of CGH modulation software, and (b) GUI of EDK experiment software. 


\subsection{Basic Characteristic of LCoS-SLM}

In this basic characteristic of LCoS-SLM, we measure the light utilization, uniformity, response time, and diffraction efficiency. The model of LCoS-SLM we use in this experiment is JD8554 from Jasper Display Corp. (JDC). This LCoSSLM is reflective type and the rubbing direction of LCoS panel is 0 degree. The resolution, active area diagonal, pixel pitch, and phase retardation is $1920 \times 1080,0.55 ”, 6.4 \mu \mathrm{m}$, and $2 \pi$ at $532 \mathrm{~nm}$, respectively. In addition, the wavelength of the coherent light source we use in these experiments is $532 \mathrm{~nm}$. First, we measure the light utilization and light utilization is defined as the following equation

$$
\text { Light Utilization }=\frac{I_{0}}{I_{\text {input }}}
$$

where $I_{\text {input }}$ is the input power and $I_{0}$ is the reflected $0^{\text {th }}$ order light power. The experiment setup of measuring the light utilization is shown in Figure 7 (a). The coherent light source whose polarization is aligned with the liquid crystal orientation hits the LCoS panel with incident angle less than 3 degree. The diameter of sensor is $9 \mathrm{~mm}$ and it is larger than the diameter of laser beam. We need to ensure all energy is collected into this sensor. We also need to keep the same distance for measuring $I_{0}$ and $I_{\text {input }}$. The light utilization is $75.9 \%$.

Second, we measure the uniformity of phase retardation. The uniformity is measured by cross polarized system, the reflectance curve is transferred to phase curved by the following formula

$$
R=\sin ^{2}\left(\frac{\theta}{2}\right)
$$

where $R$ is the reflectance, $\theta$ is the phase retardation. Figure 7 (b) shows the experiment setup of measuring the uniformity. The collimating light illuminates the LCoS-SLM. The input and output polarization are restricted by Polarization Beam Splitter (PBS) and the panel is rotated at 45 degree angles. Then, the entire LCoS-SLM data image is formed by imaging lens and is recorded by CCD. The uniformity is $3.97 \%$ at $6.3 \pi$.

Third, we measure the response time. The response time is a summation of rise time and fall time of liquid crystal. The calculation range is from $10 \%$ phase variation to $90 \%$ phase variation. We define the low driving voltage to the high driving voltage as rise time and the high driving voltage to the low driving voltage as fall time. The phase profile is obtained by the reflectance in cross polarized system as equation (2). Black and white pattern switch repeatedly in $50 \mathrm{~ms}$, and the intensity response to $2 \pi$ looks like a spike since we set the reference 0 phase at the dark sate. The experiment setup of measuring the response time is shown in Figure 7 (c) and it works as similar as the uniformity measurement system. However, we use a photo detector instead of CCD for faster time resolution. The response time includes the rise time and the fall time is $15.9 \mathrm{~ms}$. The rise time and the fall time is $4.7 \mathrm{~ms}$ and $11.2 \mathrm{~ms}$, respectively.

Finally, the diffraction efficiency is measured by N-level blazed grating, and the theoretical $1^{\text {st }}$ order diffraction efficiency of blazed grating with $2 \pi$ modulation is

$$
\text { D.E. }=\operatorname{sinc}^{2}\left(\frac{1}{N}\right)
$$

where $\mathrm{N}$ is the step number for blazed grating. For example, the binary blazed grating means that there are only two state of phase modulation, 0 and $\pi$. After the calculation, the theoretical $1^{\text {st }}$ order diffraction efficiency at the step number $\mathrm{N}=$ 2 for blazed grating is $40.6 \%$. Therefore, the theoretical value is $40.6 \%, 81.1 \%, 95.0 \%$, and $98.7 \%$ at the step number for blazed grating is $2,4,8$, and 16 , respectively. The experiment setup of measuring the diffraction efficiency is shown in Figure 7 (d). The beam splitter is used for normal illumination. The polarizer is used for controlling the input polarization to align with liquid crystal orientation. The photo detector is used for measuring the $0^{\text {th }}$ order reflected light without any grating on LCoS-SLM. This photo detector is placed at the focal plane of Fourier lens. The intensity of $0^{\text {th }}$ order reflected light is assumed as the total energy which can be modulated. Then we measure the intensity of $1^{\text {st }}$ order diffraction light with grating pattern on LCoS-SLM. The diffraction efficiency is calculated by equation (4). The measurement value is $38.8 \%, 67.7 \%, 83.0 \%$, and $92.7 \%$ at the step number for blazed grating $(\mathrm{N})$ is $2,4,8$, and 16 , respectively.

$$
D . E .=\frac{1 \text { st order diffraction light }(\text { with grating) }}{0 \text { th order reflected light (without grating) }}
$$




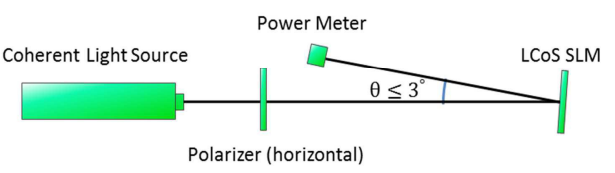

(a)

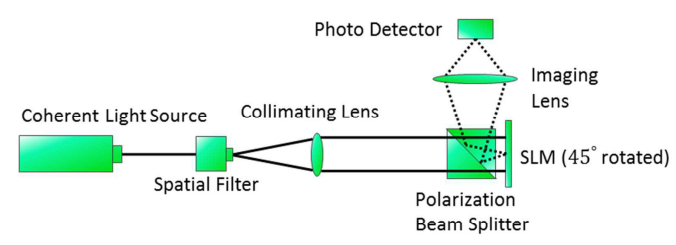

(c)

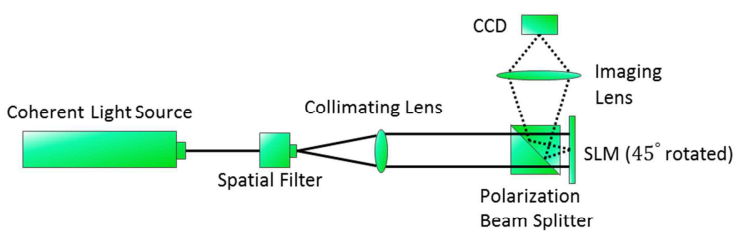

(b)

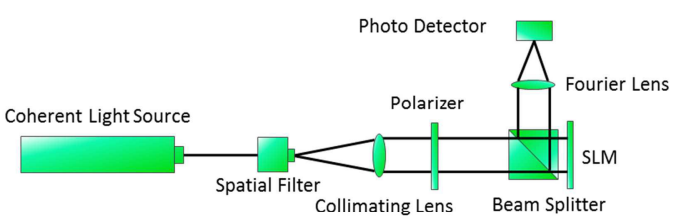

(d)

Figure 7. Experiment setup of (a) Light utilization, (b) Uniformity, (c) Response time, and (d) Diffraction efficiency.

\subsection{Wavefront Modulation on Dynamic Optics}

LCoS-SLM can receive the electrical signals to change the light signals, for example, to change the amplitude and the phase of light waves. The capability of the properly modulating light signals not only allows us to create various types of object waves in order to form the images, but also enables LCOS-SLM to modulate various kinds of simple wave forms, such as plane waves, spherical waves, cylindrical waves, Bessel beam waves, and orbital angular momentum (OAM) waves, etc. These types of wave forms are generally created by flat lenses, spherical lenses, cylindrical lenses, axicon diffractive optical element lenses, forked diffraction grating lenses, etc. Thus, being able to create these wave forms also means that LCoS-SLM can be used as adjustable optical components. The experiment setup and the diffraction image results by using CGH pattern generator and CGH modulation software are shown in Figure 8 (a). First, make sure that the laser beam runs parallel to the surface of the table by calibrating its light path. Then, install other optical components, such as lens, beam splitter, LCoS-SLM, computer, etc. Enter the CGH pattern that was generated by the CGH modulation software, then the diffraction pattern would be changed immediately. The experiment setup photo of measuring the special phase distribution is shown in Figure 8 (b).

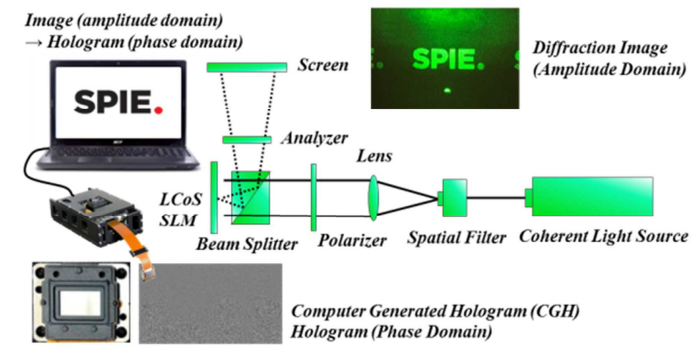

(a)

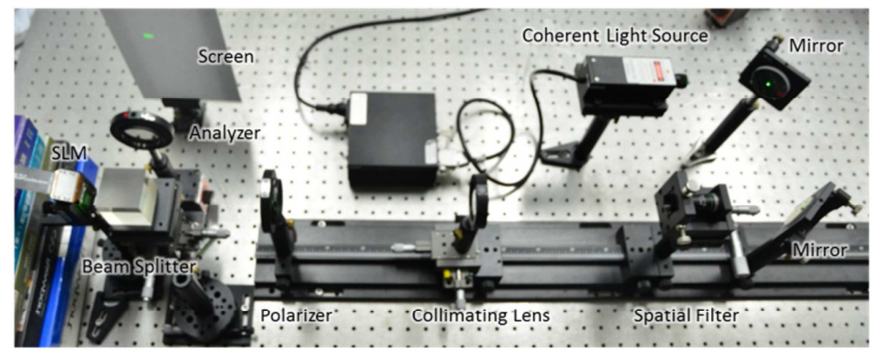

(b)

Figure 8. (a) Experiment setup and diffraction results by using CGH pattern generator and CGH modulation software, and (b)

The experiment setup photo of measuring the diffractive image.

Regarding the function of special phase distribution, this software system could provide blank phase pattern, axicon \& vortex phase pattern, binary grating phase pattern, sinusoidal grating phase pattern, and aperture phase pattern, etc. Figure 9 shows the homogeneous CGH phase pattern at different greyscale. Figure 9 (a)-(c) show the greyscale $=255$, 128 , and 0, respectively. We can also use the CGH modulation software to generate the special phase distribution, for 
example, the deflected plane wave phase pattern at specific angle, mixture of spherical and deflected plane wave phase pattern, and cylindrical wave phase pattern are shown in Figure 10 (a)-(c), respectively. The various type of wavefronts are shown on the screen, for example, not-modulated and deflected plane wave, deflected phase wave at a specific diffraction, focused convergent spherical wave at a shifted angle, and convergent cylindrical wave are shown in Figure 11(a)-(d), respectively. In addition, CGH modulation software could easy to provide the special phase distribution, such as axicon phase pattern, vortex phase pattern, and the combination of axion and vortex phase pattern as shown as Figure 12 (a)-(c), respectively. Figure 13 shows the diffraction image results at different axicon and vortex condition.

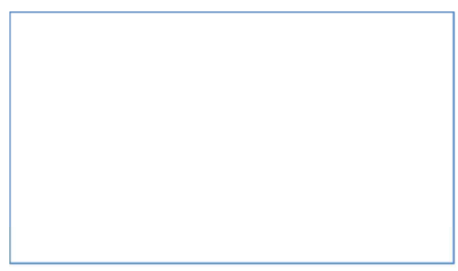

(a)

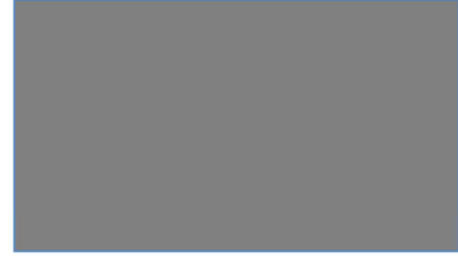

(b)

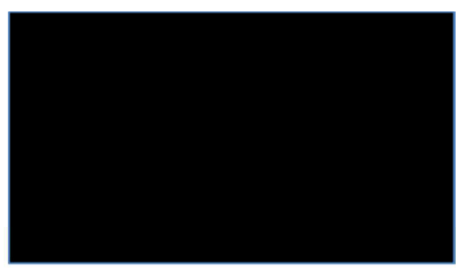

(c)

Figure 9. Homogeneous CGH phase pattern at different greyscale, (a) Greyscale $=255$, (b) Greyscale $=128$, and (c) Greyscale $=0$.

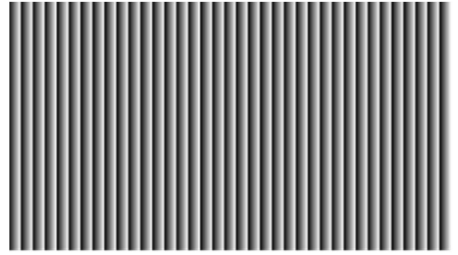

(a)

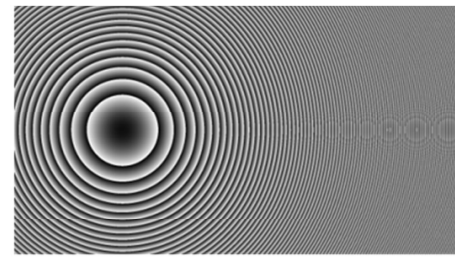

(b)

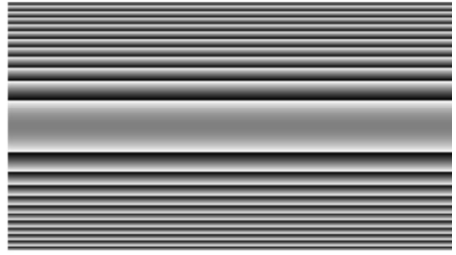

(c)

Figure 10. Special phase distribution, such as (a) Deflected plane wave phase pattern at a specific diffraction angle, (b) Mixture of spherical and deflected plane wave phase pattern, and (c) Cylindrical wave phase pattern.

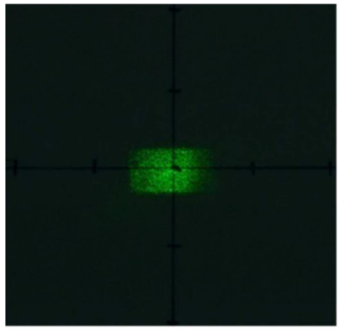

(a)

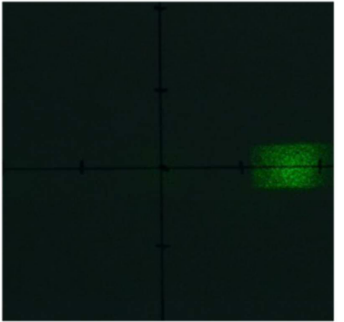

(b)

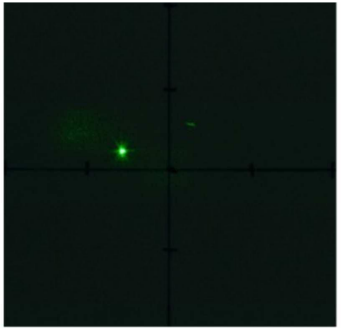

(c)

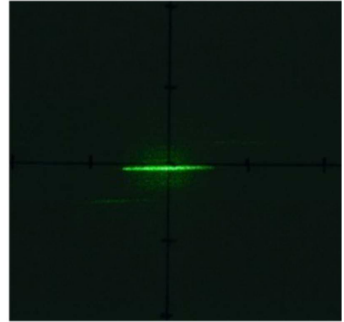

(d)

Figure 11. Various types of wavefronts on the screen, (a) Non-modulated and deflected plane wave, (b) Deflected plane wave at a specific diffraction angle, (c) Focused convergent spherical wave at a shifted angle, and (d) Convergent cylindrical wave. 


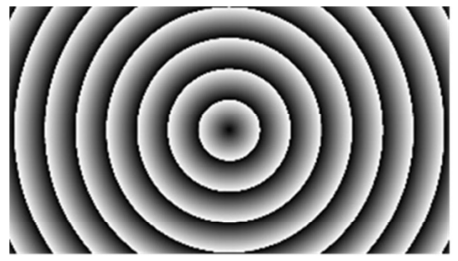

(a)

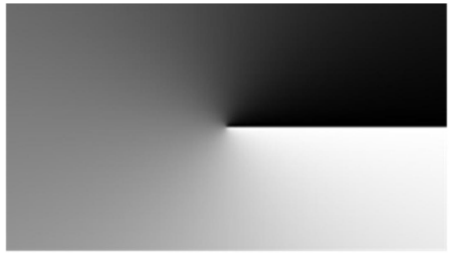

(b)

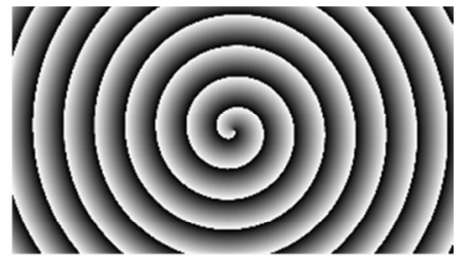

(c)

Figure 12. Special phase distribution, such as (a) Axicon phase pattern, (b) Vortex pattern, and (c) Combination of axicon and vortex phase pattern.

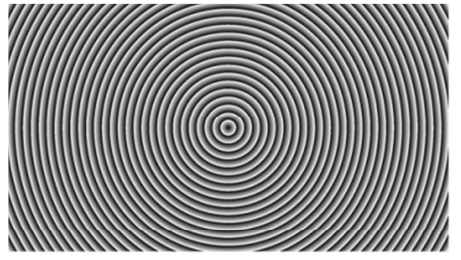

(a)

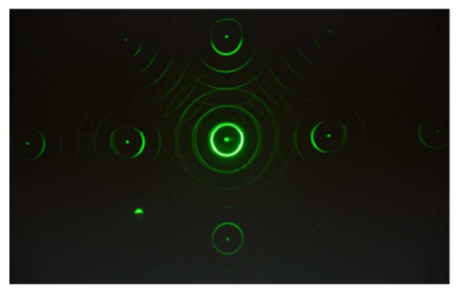

(d)

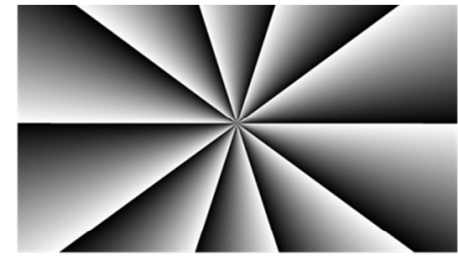

(b)

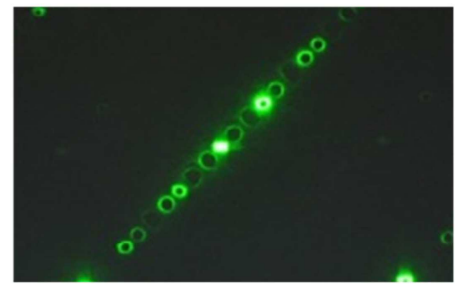

(e)

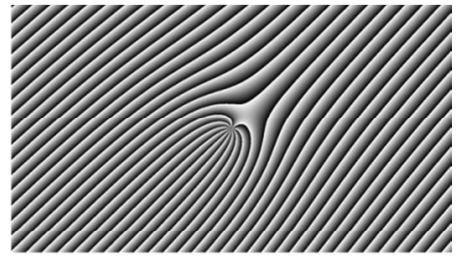

(c)

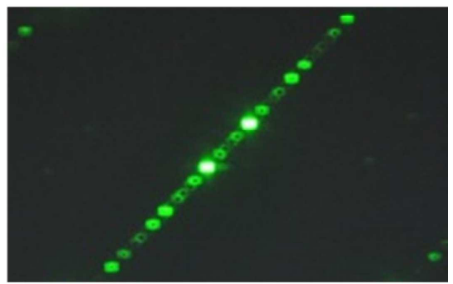

(f)

Figure 13. Diffraction image results at different axicon and vortex condition, (a) Axicon phase pattern, (b) Vortex pattern, (c) Fork pattern (vortex pattern $+x$ \& y shift pattern), (d) Diffraction image of axicon, (e) Diffraction image of fork pattern at vortex order 10, and (f) Diffraction image of fork pattern at vortex order 1.

\section{CONCLUSIONS}

In this paper, we provide a novel cooperation model to the academia, research institutions, and industries. This kind of novel cooperation model we call it as MiniFAB. We look forward to developing various applications with our partners based on our X-on Silicon technologies and the partners' domain know-how. For example, X-on Silicon technologies could be LCoS, $\mu$ LEDoS OLEDoS, CELLoS, etc. We can use this Digital Electro-optics Platform as a training kit to develop the innovation technology and to reduce the development period. Dynamic optics is one of applications by using LCoS technology. In addition, we have already developed multi-function and easy-to-use CGH modulation software and EDK experiment software. We could enhance the performance of LCoS-SLM as similar as the DOE and use it on various dynamic optics applications. Most important of all, we realized one detail that optical technology must be rooted in education to establish a solid foundation. How to find a balance between theory and practice in optics education is our mission and target. Through experiments, students become more familiar with the principles of optics, and they may find more details during experiments. This would be a great help to technology and product development when they enter industries in the future. 


\section{REFERENCES}

[1] “The International Year of Light and Light-based Technologies", http://www.light2015.org/Home.html

[2] Woerdemann, M., Holtmann, F., and Denz, C., "Holographic phase contrast for dynamic multiple-beam optical tweezers," Journal of Optics A: Pure and Applied Optics, 11(3), 034010 (2009).

[3] Li, S., Wang, Y., Lu, Z., Ding, L., Du, P., Chen, Y., Zheng, Z., Ba, D., Dong, Y., Yuan, H., Bai, Z., Liu, Z., and Cui, C., "High-quality near-field beam achieved in a high-power laser based on SLM adaptive beam-shaping system," Optics Express, 23(2), 681-689 (2015).

[4] McManamon, P.F., Bos, P.J., Escuti, M.J., Heikenfeld, J., Serati, S., Xie, H., and Watson, E.A., "A Review of Phased Array Steering for Narrow-Band Electrooptical Systems," Proceedings of IEEE, 97(6), 1078-1096 (2009).

[5] Milewski, G., Engström D., and Bengtsson, J., "Diffractive optical elements designed for highly precise farfield generation in the presence of artifacts typical for pixelated spatial light modulators," Applied Optics, 46(1), 95-105 (2007).

[6] Difato, F., Schibalsky, L., Benfenati, F., and Blau, A., "Integration of Optical Manipulation and Electrophysiological Tools to Modulate and Record Activity in Neural Networks," International Journal of Optomechatronics, 5(3), 191-216 (2011).

[7] Yao, A. M., and Padgett, M. J., "Orbital angular momentum: origins, behavior and applications," Advances in Optics and Photonics, 3(2), 161-204 (2011).

[8] Chung, C. Y., Cho, K. C., Chang, C. C., Lin, C. H., Yen, W. C., and Chen, S. J., "Adaptive-optics system with liquid-crystal phase- shift interferometer," Applied Optics, 45(15), 3409-3414 (2006).

[9] Mu, Q., "Adaptive optics imaging system based on a high-resolution liquid crystal on silicon device," Optics Express 14(18), 8013-8018 (2006)

[10] Kozacki, T., "Holographic display with tilted spatial light modulator," Applied Optics, 50(20), 3580-3588 (2011).

[11]Lin, Y. C., Tu, H. Y. and Cheng, C. J., "Characterizing spatio-temporal phase variation of pixelated liquid crystal on silicon using digital holographic microscopy," Proceeding of 2015 Digital Holography and 3D Imaging, Shanghai, China, DTh1A.5 (2015).

[12] Yang, H., Robertson, B., Wilkinson, P., and Chu, D., "Low-Cost CDC ROADM Architecture Based on Stacked Wavelength Selective Switches," Journal of Optical Communications and Networking, 9(5), 375-384 (2017).

[13] Wilkinson, T. D., "Maskless Photolithography By Holographic Optical Projection," Imaging and Applied Optics, OSA Technical Digest (online), paper DW1B.3 (2014).

[14]Li, B., Gibson, J., Middendorf, J., Wang, Y., and Zhang, S., "Comparison between LCOS projector and DLP projector in generating digital sinusoidal fringe patterns," Proceeding of SPIE 8839, Dimensional Optical Metrology and Inspection for Practical Applications II, 883908 (2013).

[15]Liu, Z J., Chong, W. C., Wong, K. M., Tam, K. H., and Lau, K. M., "A Novel BLU-Free Full-Color LED Projector Using LED on Silicon Micro-Displays," IEEE Photonics Technology Letter, 25(23), 2267-2270 (2013)

[16]Liu, Z. J. Chong, W. C., Wong, K. M., Lau, and K. M., "360 PPI Flip-Chip Mounted Active Matrix Addressable Light Emitting Diode on Silicon (LEDoS) Micro-Displays," Journal of Display Technology, 9(8), 678-682 (2013).

[17] “Jasper Display Corporation (JDC)", http://www.jasperdisplay.com

[18] "glō", http://www.glo.se

[19] Tsai, C. W., Lyu, B. H., Wang, C., and Hung, C. C., "Enhancing performance of LCoS-SLM as adaptive optics by using computer-generated holograms modulation software," Proceeding of SPIE 10233, Holography: Advances and Modern Trends, Prague, Czech Republic, 1023347 (2017).

[20] Wang, C., Hsu, Y. C., and Chan, S. H., "SLM-based education kit for wave optics," Proceeding of SPIE 9793, Education and Training in Optics and Photonics: ETOP 2015, Bordeaux, France, 979315 (2015).

[21] "EDK Application Software by Jasper Display" at LabVIEW Tools Network of National Instruments (NI) website, http://sine.ni.com/nips/cds/view/p/lang/en/nid/215468 